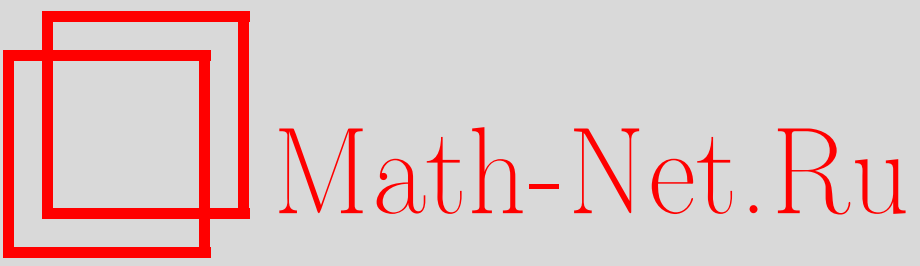

И. Т. Хабибуллин, Дискретные цепочки серии С, ТМФ, 2006, том 146, номер 2, 208-221

DOI: https://doi.org/10.4213/tmf2032

Использование Общероссийского математического портала Math-Net.Ru подразумевает, что вы прочитали и согласны с пользовательским соглашением

http://www . mathnet.ru/rus/agreement

Параметры загрузки:

IP : 3.85 .5 .30

26 апреля 2023 г., $14: 32: 43$ 


\section{ДИСКРЕТНЫЕ ЦЕПОЧКИ СЕРИИ С}

Найдены аналоги обобщенных двумеризованных цепочек Тоды серий $C_{N}$ и $\widetilde{C}_{N}$ с тремя дискретными независимыми переменными. Предъявлены пары Лакса для найденных цепочек.

Ключевые слова: дискретные цепочки Тоды, интегрируемые краевые условия, дискретные гиперболические уравнения, инварианты Лапласа, пара Лакса, простые алгебры Ли.

\section{1. ВВЕДЕНИЕ}

В данной работе рассматривается цепочка Тоды с дискретным временем [1], [2]

$$
e^{f_{u v}-f_{u}-f_{v}+f}=\frac{1+h e^{f_{v}^{1}-f_{u}}}{1+h e^{f_{v}-f_{u}^{-1}}} .
$$

Здесь искомая функция $f=f(u, v, k)$ зависит от трех независимых дискретных переменных. Верхний индекс у функции указывает на сдвиг по переменной $k$ так, что $f^{1}=f(u, v, k+1)$ и $f^{-1}=f(u, v, k-1)$. Нижний индекс обозначает сдвиги по первому и второму аргументу: $f_{u}=f(u+1, v, k), f_{-u}=f(u-1, v, k), f_{v}=f(u, v+$ $1, k), f_{-v}=f(u, v-1, k)$. Параметр $h$ соответствует шагу сетки, при малых $h$ имеем $f_{u}-f \simeq \sqrt{h} D_{x} f$, а также $f_{v}-f \simeq \sqrt{h} D_{y} f$ и $f_{u v}-f_{u}-f_{v}+f \simeq h D_{x} D_{y} f$, где $D_{x}$ и $D_{y}$ - операторы дифференцирования по $x$ и $y$. Ясно, что в континуальном пределе при $h \rightarrow 0$ дискретная цепочка Тоды (ДЦТ) (1) переходит в обычную двумеризованную цепочку Тоды [3]

$$
D_{x} D_{y} f=e^{f^{1}-f}-e^{f-f^{-1}} .
$$

ДЦТ тесно связана с дискретным билинейным уравнением Хироты-Мивы

$$
t t_{u v}-t_{u} t_{v}=t_{v}^{1} t_{u}^{-1}
$$

Здесь искомая функция $t=t(u, v, k)$ также зависит от трех переменных. Преобразование типа Миуры $t^{1}=e^{f} t$ переводит уравнение (3) в уравнение (1).

* Институт математики Уфимского научного центра РАН, Уфа, Россия. E-mail: ihabib@imat.rb.ru 
Цепочка (1) допускает, очевидно, периодическое замыкание $f(u, v, k)=f(u, v, k+$ $N)$, приводящее ее к конечно-полевому уравнению с динамическими переменными $f(u, v, 1), f(u, v, 2), \ldots, f(u, v, N)$. Другая простая конечно-полевая редукция связана с точкой вырождения уравнения; например, краевые условия вида $e^{-f(u, v, 0)}=0$ и $e^{f(u, v, N+1)}=0$ также сводят цепочку (1) к интегрируемой системе разностных гиперболических уравнений. Но, как правило, интегрируемые редукции цепочек не исчерпываются периодическим и вырожденным случаями. Известно, например, что двумеризованная цепочка Тоды (2) допускает широкий класс редукций, связанных с простыми алгебрами Ли и алгебрами Каца-Муди [4]. Упоминавшиеся выше вырожденная и периодическая редукции соответствуют алгебрам серий $A_{n}$ и $\tilde{A}_{n}$. Проблема поиска дискретных аналогов конечно-полевых цепочек Тоды (2), соответствующих остальным алгебрам Ли конечного роста, остается открытой и вызывает определенный интерес (см., например, [5], а также обзоры [6]). В работе [5] обсуждались примеры обрывов, связанных со специальными решениями цепочки (1). Ниже мы предлагаем дискретные аналоги для цепочек Тоды серий $C_{N}, \widetilde{C}_{N}$.

Основными результатами настоящей работы являются следующие два предложения.

ПРЕДЛОЖЕНИЕ 1. Конечно-полевая чепочка вида

$$
\begin{aligned}
f_{u,-v}^{-2} & =f^{-1}+f-f_{-u, v}^{1}, \\
e^{f_{u v}^{j}-f_{u}^{j}-f_{v}^{j}+f^{j}} & =\frac{1+h e^{f_{v}^{j+1}-f_{u}^{j}}}{1+h e^{f_{v}^{j}-f_{u}^{j-1}}} \quad \text { npu } \quad-1 \leqslant j \leqslant N, \\
e^{f^{N+1}} & =0
\end{aligned}
$$

допускает пару Лакса (см. Формулы (54), (55) ниже) и в континуальном пределе nри $h \rightarrow 0$ переходит в конечную двумеризованную цепочку Тоды серии $C_{N}$

$$
\begin{gathered}
u(-1)=-u(0), \\
u_{x y}=e^{u(k+1)-u(k)}-e^{u(k)-u(k-1)}, \\
e^{u(N+1)}=0 .
\end{gathered}
$$

ПРЕДЛОЖЕНИЕ 2. Конечно-полевая чепочка вида

$$
\begin{aligned}
f_{u,-v}^{-2} & =f^{-1}+f-f_{-u, v}^{1}, \\
e^{f_{u v}^{j}-f_{u}^{j}-f_{v}^{j}+f^{j}} & =\frac{1+h e^{f_{v}^{j+1}-f_{u}^{j}}}{1+h e^{f_{v}^{j}-f_{u}^{j-1}}} \quad n p u \quad-1 \leqslant j \leqslant N, \\
f_{-u, v}^{N+1} & =f^{N}+f^{N-1}-f_{u,-v}^{N-2},
\end{aligned}
$$

допускает пару Лакса (см. Формулы (61), (62) ниже) и в континуальном пределе nри $h \rightarrow 0$ переходит в конечную двумеризованную цепочку Тоды серии $\widetilde{C}_{N}$

$$
\begin{aligned}
u(-1) & =-u(0), \\
u_{x y} & =e^{u(k+1)-u(k)}-e^{u(k)-u(k-1)}, \\
u(N) & =-u(N-1) .
\end{aligned}
$$


Для сокращения формул мы будем пользоваться следующими обозначениями:

$$
z(u, v, k)=1+h e^{f_{v}-f_{u}^{-1}}, \quad \Delta f=f_{u v}-f_{u}-f_{v}+f,
$$

тогда цепочка (1) примет вид $z^{1}=e^{\Delta f} z$. Не ограничивая общности, можно считать, что $h=1$. Действительно, значение $h=1$ можно получить при помощи преобразования сдвига $f(k) \rightarrow f(k)+k \ln h$.

Обсудим кратко содержание работы. В разделе 2 показано, что гиперболическое уравнение (9), вытекающее из пары Лакса, удовлетворяет некоторому условию инволюции (точнее говоря, это уравнение связано с двойственным к нему уравнением (11) мультипликативной заменой) при фиксированном значении $k=k_{0}$ тогда и только тогда, когда выполняется следующее условие редукции: $z_{u}^{k_{0}-1}=z_{v}^{k_{0}+1}$. Далее, в разделе 3 проверяется, что это условие редукции определяет обрыв цепочки (1), сохраняющий интегрируемость. В переменных $f$ условие обрыва имеет вид $f_{u,-v}^{k_{0}-2}=f^{k_{0}-1}+f^{k_{0}}-f_{-u, v}^{k_{0}+1}$. В пределе при $h \rightarrow 0$ это условие (с точностью до несущественных точечных преобразований) переходит в условие обрыва $f^{k_{0}-1}=-f^{k_{0}}$ для цепочки (2). Пары Лакса для конечных цепочек выписаны в разделе 4. Отметим характерную особенность пар Лакса для конечных цепочек: наряду со сдвигами вперед по переменным $u$ и $v$ они содержат также сдвиги назад.

\section{2. ИНВОЛЮЦИИ АССОЦИИРОВАННОГО ЛИНЕЙНОГО ГИПЕРБОЛИЧЕСКОГО УРАВНЕНИЯ}

Цепочка (1) допускает пару Лакса, задаваемую в виде системы дискретных линейных уравнений [1]

$$
\psi_{u}=e^{f_{u}-f} \psi-\psi^{1}, \quad \psi_{v}=\psi+e^{f_{v}-f^{-1}} \psi^{-1}
$$

Здесь для индексов собственной функции $\psi=\psi(u, v, k)$ приняты те же обозначения, что и для полевых переменных: $\psi_{u}=\psi(u+1, v, k), \quad \psi_{v}=\psi(u, v+1, k), \quad \psi^{1}=$ $\psi(u, v, k+1), \psi^{-1}=\psi(u, v, k-1)$ и т.д.

Исключим из системы уравнений (8) сдвиги по третьей переменной. В результате получим линейное дискретное гиперболическое уравнение

$$
\psi_{u v}-\psi_{u}-e^{f_{u v}-f_{v}}\left(\psi_{v}-z \psi\right)=0
$$

(напомним, что $z=1+e^{f_{v}-f_{u}^{-1}}$ ).

Ниже нам понадобятся также уравнения, двойственные к (8) и (9). Для того чтобы найти двойственные уравнения, можно воспользоваться дискретными симметриями цепочки (1). Например, цепочка инвариантна относительно инволюции $u \rightarrow 1-v, \quad v \rightarrow 1-u$. Более точно, рассматривается замена переменной вида $f=f(u, v) \rightarrow f(1-v, 1-u)=f_{u v}(-v,-u)$. При такой замене имеем

$$
\begin{aligned}
& f_{u}=f(u+1, v) \rightarrow f(1-v, 1-(u+1))=f_{u}(-v,-u), \\
& f_{v}=f(u, v+1) \rightarrow f(1-(v-1), 1-u)=f_{v}(-v,-u), \quad f_{u v} \rightarrow f(-v,-u) .
\end{aligned}
$$


Прямой подстановкой легко убедиться, что цепочка не изменяется при такой замене. И в то же время замена переменных меняет пару Лакса (8), которая принимает вид

$$
y_{-u}=y+e^{f_{-u}-f^{-1}} y^{-1}, \quad y_{-v}=e^{f_{-v}-f} y-y^{1},
$$

где $y(u, v)=\psi(1-v, 1-u)$, причем гиперболическое уравнение (9) переходит в уравнение вида

$$
y_{u v}-\frac{1}{z} y_{v}-\frac{e^{f_{v}-f}}{z}\left(y_{u}-y\right)=0
$$

поскольку $z \rightarrow z$.

Ясно, что это не единственный способ выбора двойственного уравнения. Например, можно в качестве двойственной пары Лакса взять сопряженную пару. Но это мало отразится на окончательном ответе, так как сопряженная пара калибровочно эквивалентна паре (10). Автору не удалось найти двойственную пару Лакса, которая не была бы калибровочно эквивалентна ни одной из пар (8), (10).

Отметим, что идея использовать одновременно две (взаимно сопряженные) пары Лакса при изучении конечных цепочек Тоды восходит к классической работе Дарбу [3].

Действуя по аналогии с обычной двумеризованной цепочкой Тоды, выясним, при каких ограничениях на коэффициенты гиперболические уравнения (9) и (11) связаны между собой мультипликативной заменой вида $y=a \psi$ (см. [7]). Как известно, необходимым и достаточным условием наличия мультипликативной связи между двумя гиперболическими уравнениями является совпадение инвариантов Лапласа этих уравнений [8]. Напомним, что инварианты Лапласа уравнения

$$
a \psi+b \psi_{u}+c \psi_{v}+d \psi_{u v}=0
$$

имеют вид

$$
K_{1}=\frac{b c_{u}}{d a_{u}}, \quad K_{2}=\frac{b_{v} c}{d a_{v}} .
$$

Обозначим через $K_{1 \psi}(u, v, k), K_{2 \psi}(u, v, k)$ инварианты Лапласа уравнения (9), а через $K_{1 y}(u, v, k), K_{2 y}(u, v, k)$ - инварианты Лапласа уравнения (11). Вычислив все эти инварианты, получим

$$
K_{1 y}=\frac{1}{z^{1}}, \quad K_{2 y}=\frac{1}{z}, \quad K_{1 \psi}=\frac{1}{z_{u}}, \quad K_{2 \psi}=\frac{1}{z_{v}^{1}} .
$$

Отсюда видно, что если для некоторого фиксированного значения $k=k_{0}$ наложить на цепочечные переменные следующее условие связи:

$$
z\left(u+1, v, k_{0}-1\right)=z\left(u, v+1, k_{0}+1\right),
$$

то инварианты Лапласа двух рассматриваемых уравнений будут удовлетворять условиям

$$
K_{1 y}\left(u+1, v, k_{0}-1\right)=K_{1 \psi}\left(u, v, k_{0}\right), \quad K_{2 y}\left(u+1, v, k_{0}-1\right)=K_{2 \psi}\left(u, v, k_{0}\right)
$$


а также условиям

$$
K_{1 \psi}\left(u, v-1, k_{0}-1\right)=K_{1 y}\left(u, v, k_{0}\right), \quad K_{2 \psi}\left(u, v-1, k_{0}-1\right)=K_{2 y}\left(u, v, k_{0}\right) .
$$

Следовательно, существуют такие функции $R=R(u, v), \quad S=S(u, v)$, что выполняются условия связи

$$
\psi\left(u, v-1, k_{0}-1\right)=R y\left(u, v, k_{0}\right), \quad y\left(u+1, v, k_{0}-1\right)=S \psi\left(u, v, k_{0}\right)
$$

между решениями гиперболических уравнений (9) и (11). Функции $R$ и $S$ определяются как решения следующей переопределенной системы линейных уравнений:

$$
\begin{aligned}
R_{u} & =R z_{-v} e^{f_{u,-v}^{-1}-f_{-v}^{-1}}, & R_{v} & =R z_{-u} e^{f_{-u}-f_{-u, v},}, \\
S_{u} & =S \frac{e^{f-f_{u}}}{z_{-u,-v}^{-1}}, & S_{v} & =S \frac{e^{f_{v}^{-1}-f^{-1}}}{z^{-1}},
\end{aligned}
$$

совместность которой гарантируется условием (15).

\section{3. ПАРА ЛАКСА ДЛЯ ПОЛУБЕСКОНЕЧНОЙ ЦЕПОЧКИ}

Равенства (18) фактически являются краевыми условиями, замыкающими линейные системы (8), (10) на полуось $k \geqslant k_{0}$ (либо на полуось $k \leqslant k_{0}$ ). Поясним и докажем это утверждение. Для сокращения формул временно положим $k_{0}=0$. Подставляя (18) в уравнения (8) и (10) при $k=0$, находим

$$
y_{-u}^{0}=y^{0}+X_{-u} \psi_{-u}^{0}, \quad \psi_{v}^{0}=\psi^{0}+H_{v} y_{v}^{0}
$$

где $X=S e^{f-f_{u}^{-1}}, H=R e^{f-f_{-v}^{-1}}$.

Справедлива следующая лемма, связывающая функции $X$ и $H$.

Лемма 1. Решения системы уравнений (19), (20) можно выбрать так, чтобъ выполнялось равенство

$$
H X=\frac{z_{-v}-1}{z_{-v}}
$$

ДокАЗАТЕЛЬСтво. Из уравнений (19) и (20) легко можно вывести, что $X$ и $H$ удовлетворяют аналогичным линейным разностным уравнениям первого порядка

$$
\begin{array}{ll}
X_{v}=X \frac{e^{f_{v}-f}}{z}, & H_{v}=H z_{-u}^{1} e^{f_{-v}^{-1}-f^{-1}}, \\
X_{u}=X \frac{e^{f_{u}^{-1}-f_{u^{2}}^{-1}}}{z^{1}}, & X_{v}=X \frac{e^{f_{v}-f}}{z},
\end{array}
$$

где $f_{u^{2}}^{-1}:=f(u+2, v, k-1)$.

Простой расчет показывает, что если в некоторой точке $(u, v)$ выполняется равенство (22), то оно выполняется и в соседних точках. Лемма доказана. 
Всюду ниже будет предполагаться, что условие (22) выполняется.

Кратко обсудим вопрос о динамических переменных. Легко видеть, что сдвиги $\psi_{u}, \psi_{u^{2}}, \psi_{v}, \psi_{v^{2}}, \ldots$ собственной функции $\psi$ в положительном направлении, также как и сдвиги $y_{-u}, y_{-u^{2}}, y_{-v}, y_{-v^{2}}, \ldots$ собственной функции $y$ в отрицательном направлении, локально выражаются через несдвинутые (динамические) переменные $\psi$, $\psi^{ \pm 1}, \psi^{ \pm 2}, \ldots$ и $y, y^{ \pm 1}, y^{ \pm 2}, \ldots$. Другое дело - сдвиги в противоположных направлениях, которые можно рассматривать как нелокальные переменные. Действительно, эти переменные невозможно выразить через конечное число динамических переменных. Например, для того чтобы выразить $\psi_{-u}$ через $\psi$, нужно решить разностное (по аргументу $k$ ) уравнение вида

$$
\psi_{-u}^{1}-e^{f-f_{-u}} \psi_{-u}=\psi
$$

Расширим динамический набор переменных. В дополнение к динамическому набору собственных функций на полуоси $k \geqslant 0$, состоящему из функций $\left\{\psi^{0}, y^{0}, \psi^{1}, y^{1}, \ldots\right\}$, введем еще две переменные $Y$ и $\Psi$, полагая $Y=y_{v}^{0}, \Psi=\psi_{-u}^{0}$.

ЛЕмма 2. Сдвиги $Y_{-u}, Y_{-v}, Y_{u}$ переменной $Y$ и сдвиги $\Psi_{u}, \Psi_{v}, \Psi_{-v}$ переменной $\Psi$ линейно выражаются через конечное число функиий из расширенного набора динамических переменных $\left\{\Psi, Y, \psi^{0}, y^{0}, \psi^{1}, y^{1}, \ldots\right\}$.

ДокАЗАТЕЛЬСтво. Непосредственно из определения имеем часть требуемых равенств: $Y_{-v}=y^{0}, \Psi_{u}=\psi^{0}$. Сдвинем первое уравнение $(21)$ на единицу вперед по $u$ и по $v$ и перепишем его в виде

$$
Y_{u}=Y-X_{v} \psi_{v}^{0}=Y-X_{v}\left(\psi^{0}+H_{v} Y\right)=Y\left(1-X_{v} H_{v}\right)-X_{v} \psi^{0} .
$$

Воспользовавшись леммой 1 , запишем равенство $1-X_{v} H_{v}=z^{-1}$, отсюда

$$
Y_{u}=\frac{1}{z} Y-X_{v} \psi^{0}
$$

Сдвинем полученное выражение на единицу по $u$ назад и преобразуем его к виду

$$
Y_{-u}=z_{-u} Y+X_{-u, v} z_{-u} \Psi
$$

Сдвинем теперь второе уравнение (21) на единицу назад по $u$ и по $v$ и в результате несложных вычислений получим

$$
\begin{aligned}
\Psi_{-v} & =\frac{1}{z_{-u,-v}} \Psi-H_{-u} y^{0}, \\
\Psi_{v} & =z_{-u} \Psi+H_{-u, v} z_{-u} Y .
\end{aligned}
$$

Прокомментируем лемму 2. Переменные $\psi^{j}$ можно сдвигать вперед, а переменные $y^{j}$ - назад по $u$ и $v$. Новые динамические переменные занимают промежуточное положение, их можно сдвигать в трех направлениях. 
Подведем итог вычислениям, проделанным выше. Введем некоторые обозначения. Обозначим через $P$ и $Q$ бесконечномерные векторы-столбцы

$$
P=\left(\begin{array}{c}
Y \\
y^{0} \\
y^{1} \\
y^{2} \\
\ldots
\end{array}\right), \quad Q=\left(\begin{array}{c}
\Psi \\
\psi^{0} \\
\psi^{1} \\
\psi^{2} \\
\ldots
\end{array}\right),
$$

т.е. $P_{0}=Y, Q_{0}=\Psi$ и $P_{i}=y^{i-1}, Q_{i}=\psi^{i-1}$ при $i \geqslant 1$. Введем в рассмотрение шесть бесконечномерных матриц, из которых четыре имеют двухдиагональный вид:

$$
\begin{aligned}
A & =\left(\begin{array}{ccccc}
z_{u} & 0 & 0 & 0 & \ldots \\
0 & 1 & 0 & 0 & \ldots \\
0 & e^{f_{-u}^{1}-f} & 1 & 0 & \ldots \\
0 & 0 & e^{f_{-u}^{2}-f^{1}} & 1 & \ldots \\
\ldots & \ldots & \ldots & \ldots & \ldots
\end{array}\right) \\
B & =\left(\begin{array}{cccccc}
0 & 1 & 0 & 0 & \ldots \\
0 & e^{f_{-v}-f} & -1 & 0 & \ldots \\
0 & 0 & e^{f_{-v}^{1}-f^{1}} & -1 & \ldots \\
0 & 0 & 0 & e^{f_{-v}^{2}-f^{2}} & \ldots \\
\ldots & \ldots & \ldots & \ldots & \ldots
\end{array}\right), \\
C & =\left(\begin{array}{cccccc}
z_{v} & 0 & 0 & 0 & \ldots \\
0 & 1 & 0 & 0 & \ldots \\
0 & e^{f_{v}^{1}-f} & 1 & 0 & \ldots \\
0 & 0 & e^{f_{v}^{2}-f^{1}} & 1 & \ldots \\
\ldots & \ldots & \ldots & \ldots & \ldots
\end{array}\right), \\
D & =\left(\begin{array}{cccccc}
0 & 1 & 0 & 0 & \ldots \\
0 & e^{f_{u}-f} & -1 & 0 & \ldots \\
0 & 0 & e^{f_{u}^{1}-f^{1}} & -1 & \ldots \\
0 & 0 & 0 & e^{f_{u}^{2}-f^{2}} & \ldots \\
\ldots & \ldots & \ldots & \ldots & \ldots
\end{array}\right)
\end{aligned}
$$

и две сильно разреженные:

$$
a=\left(\begin{array}{ccc}
X_{-u, v} z_{-u} & 0 & \ldots \\
X_{-u} & 0 & \ldots \\
0 & 0 & \ldots \\
\ldots & \ldots & \ldots
\end{array}\right), \quad c=\left(\begin{array}{ccc}
H_{-u, v} z_{v} & 0 & \ldots \\
H_{v} & 0 & \ldots \\
0 & 0 & \ldots \\
\ldots & \ldots & \ldots
\end{array}\right)
$$

У последних двух матриц все элементы равны нулю, за исключением $a_{0,0}, a_{1,0}$, $c_{0,0}, c_{1,0}$.

Составим систему уравнений в сдвигах:

$$
\begin{aligned}
P_{-u} & =A P+a Q, & P_{-v} & =B P, \\
Q_{v} & =C Q+c P, & Q_{u} & =D Q .
\end{aligned}
$$


ПРЕДЛОЖеНИЕ 3. Система уравнений (31), (32) совместна тогда и только тогда, когда ее коэффициенты удовлетворяют иепочке уравнений

$$
\begin{aligned}
e^{\Delta f^{-1}} & =\frac{z}{z_{-u, v}^{1}}, \\
e^{\Delta f^{j}} & =\frac{z^{j+1}}{z^{j}} \quad \text { npu } \quad 0 \leqslant j<\infty .
\end{aligned}
$$

Для доказательства предложения достаточно проверить, что равенства $\left(P_{-u}\right)_{-v}=$ $\left(P_{-v}\right)_{-u},\left(Q_{u}\right)_{v}=\left(P_{v}\right)_{u}$ выполняются только при условиях (33), (34). Ясно, что система уравнений $(31),(32)$ есть матричная запись следующей системы уравнений:

$$
\begin{array}{rlrl}
\psi_{u}^{j} & =e^{f_{u}^{j}-f^{j}} \psi^{j}-\psi^{j+1}, & y_{-v}^{j}=e^{f_{-u}^{j}-f^{j}} y^{j}-y^{j+1} \quad \text { при } \quad j \geqslant 0, \\
\psi_{v}^{j}=\psi^{j}+e^{f_{v}^{j}-f^{j-1}} \psi^{j-1}, & y_{-u}^{j}=y^{j}+e^{f_{-u}^{j}-f^{j-1}} y^{j-1} \quad \text { при } j \geqslant 1, \\
\psi_{v}^{0}=\psi^{0}+H_{v} y_{v}^{0}, & y_{-u}^{0}=y^{0}+X_{-u} \psi_{-u}^{0}, \\
\Psi_{v}=z_{-u} \Psi+H_{-u, v} z_{-u} Y, & Y_{-u}=z_{-u} Y+X_{-u, v} z_{-u} \Psi, & \\
\Psi_{u}=\psi^{0}, & Y_{-v}=y^{0} .
\end{array}
$$

Другими словами, нужно проверить выполнение равенств

$$
\left(Y_{-u}\right)_{-v}=\left(Y_{-v}\right)_{-u}, \quad\left(\Psi_{u}\right)_{v}=\left(\Psi_{v}\right)_{u}, \quad\left(y_{-u}^{j}\right)_{-v}=\left(y_{-v}^{j}\right)_{-u}, \quad\left(\psi_{u}^{j}\right)_{v}=\left(\psi_{v}^{j}\right)_{u},
$$

причем достаточно взять $j=0$, так как для других $j$ эти равенства заведомо выполнены. Можно проверить непосредственно, что требуемые равенства равносильны следующим пяти условиям:

$$
\begin{gathered}
X_{v}=X \frac{e^{f_{v}-f}}{z}, \quad z^{1} e^{-\Delta f}=1+(z-1) \frac{z_{-u}^{1}}{z_{-v}^{-1}}, \quad X_{v}=X \frac{e^{f_{v}-f}}{z}, \\
z^{1}=z e^{\Delta f}, \quad H X=\frac{z_{-v}-1}{z_{-v}} .
\end{gathered}
$$

Второе и четвертое из указанных условий приводят к краевому условию вида $z_{v}^{1}=$ $z_{u}^{-1}$, а остальные, как было показано выше, непротиворечивы при выполнении краевого условия.

Для выполнения предельного перехода при $h \rightarrow 0$ запишем краевое условие $z_{v}^{1}=$ $z_{u}^{-1}$ в развернутом виде

$$
f_{u,-v}^{-2}=f^{-1}+f-f_{-u, v}^{1}
$$

Поскольку $f_{u}=f+h D_{x} f+O\left(h^{2}\right)$ и $f_{v}=f+h D_{y} f+O\left(h^{2}\right)$, то в пределе при $h \rightarrow 0$ получим $f^{-2}=f^{-1}+f-f^{1}$. Тогда из уравнения (2) легко получить, что $D_{x} D_{y}(f+$ $\left.f^{-1}\right)=0$. Отсюда имеем $f=-f_{-1}+a(x)+b(y)$. Уберем произвол, связанный с функциями $a(x), b(y)$, при помощи точечного преобразования $f=\tilde{f}+a(x) / 2+b(y) / 2$ и придем к искомому условию обрыва $f^{-1}=-f$.

Отметим, что при дополнительном ограничении $f(u, v, k)=f(u+v, k)$, наложенном на цепочку $(1)$, мы приходим к $(1+1)$-мерной дискретной цепочке Тоды. Тогда 
граничное условие (40) переходит в условие $f(u,-1)=-f(u, 0)$, найденное Сурисом в работе [9] (см. также [10]).

Рассмотрим теперь другую половину цепочки, расположенную на левой полуоси $k \leqslant k_{0}$. Формулы (18) позволяют исключить из пары Лакса функции $y^{0}$ и $\psi^{0}$ и переписать сдвиги $y_{-v}^{-1}$ и $\psi_{u}^{-1}$ в виде

$$
y_{-v}^{-1}=y^{-1} e^{f_{-v}^{-1}-f^{-1}}-\frac{1}{R} \psi_{-v}^{-1}, \quad \psi_{u}^{-1}=\psi^{-1} e^{f_{u}^{-1}-f^{-1}}-\frac{1}{S} y_{u}^{-1} .
$$

Введем новые дополнительные динамические переменные $\Psi^{-1}:=\psi_{-v}^{-1}, \quad Y^{-1}:=y_{u}^{-1}$. Тогда предыдущее уравнение можно переписать так:

$$
y_{-v}^{-1}=y^{-1} e^{f_{-v}^{-1}-f^{-1}}-\frac{1}{R} \Psi^{-1}, \quad \psi_{u}^{-1}=\psi^{-1} e^{f_{u}^{-1}-f^{-1}}-\frac{1}{S} Y^{-1} .
$$

Далее нам понадобится следующий аналог леммы 1.

Лемма 3. Решения уравнений (19), (20) можно выбрать так, чтобъ выполнялось соотношение

$$
R_{u v} S=\frac{z}{z-1}
$$

Для доказательства леммы достаточно выразить $H$ и $X$ через $R$ и $S$ и подставить в формулы (22).

Лемма 4. Сдвиги $Y_{-u}^{-1}, Y_{-v}^{-1}, Y_{v}^{-1}, \Psi_{u}^{-1}, \Psi_{v}^{-1}, \Psi_{-u}^{-1}$ переменнъх $Y^{-1} u \Psi^{-1}$ линейно выражаются через конечное число функций из расширенного набора динамических переменных $\left\{\Psi^{-1}, Y^{-1}, \psi^{-1}, y^{-1}, \psi^{-2}, y^{-2}, \ldots\right\}$ :

$$
\begin{aligned}
& Y_{-u}^{-1}=y^{-1}, \quad Y_{v}^{-1}=\frac{1}{z} e^{f_{u v}^{-1}-f_{u}^{-1}} Y^{-1}+\frac{1}{R_{u v}} e^{f_{u v}^{-1}-f^{-1}} \psi^{-1}, \\
& Y_{-v}^{-1}=z_{-v} e^{f_{u,-v}^{-1}-f_{u}^{-1}} Y^{-1}-\frac{z_{-v}}{R_{u}} e^{f_{u,-v}^{-1}-f_{-v}^{-1}} \Psi^{-1} \\
& \Psi_{-u}^{-1}=\frac{1}{z_{-u,-v}} e^{f_{-u,-v}^{-1}-f_{-v}^{-1}} \Psi^{-1}+\frac{1}{S_{-u,-v}} e^{f_{-u,-v}^{-1}-f^{-1}} y^{-1}, \\
& \Psi_{v}^{-1}=\psi^{-1}, \quad \Psi_{u}^{-1}=z_{-v} e^{f_{u,-v}^{-1}-f_{-v}^{-1}} \Psi^{-1}-\frac{z_{-v}}{S_{-v}} e^{f_{u,-v}^{-1}-f_{u}^{-1}} Y^{-1} .
\end{aligned}
$$

Лемма доказывается аналогично лемме 2.

Введем бесконечные векторы-столбцы

$$
\widetilde{P}=\left(\begin{array}{c}
\cdots \\
y^{-3} \\
y^{-2} \\
y^{-1} \\
Y^{-1}
\end{array}\right), \quad \widetilde{Q}=\left(\begin{array}{c}
\cdots \\
\psi^{-3} \\
\psi^{-2} \\
\psi^{-1} \\
\Psi^{-1}
\end{array}\right)
$$


и бесконечномерные матрицы

$$
\begin{aligned}
& \tilde{A}=\left(\begin{array}{ccccc}
\cdots & \cdots & \cdots & \cdots & \cdots \\
\cdots & 1 & 0 & 0 & 0 \\
\cdots & e^{f_{-u}^{-2}-f^{-3}} & 1 & 0 & 0 \\
\cdots & 0 & e^{f_{-u}^{-1}-f^{-2}} & 1 & 0 \\
\cdots & 0 & 0 & 1 & 0
\end{array}\right) \\
& \widetilde{B}=\left(\begin{array}{ccccc}
\cdots & \cdots & \cdots & \cdots & \cdots \\
\cdots & e^{f_{-v}^{-3}-f^{-3}} & -1 & 0 & 0 \\
\cdots & 0 & e^{f_{-v}^{-2}-f^{-2}} & -1 & 0 \\
\cdots & 0 & 0 & e^{f_{-v}^{-1}-f^{-1}} & 0 \\
\cdots & 0 & 0 & 0 & z_{-v} e^{f_{u,-v}^{-1}-f_{u}^{-1}}
\end{array}\right) \\
& \widetilde{C}=\left(\begin{array}{ccccc}
\cdots & \cdots & \cdots & \cdots & \cdots \\
\cdots & 1 & 0 & 0 & 0 \\
\cdots & e^{f_{v}^{-2}-f^{-3}} & 1 & 0 & 0 \\
\cdots & 0 & e^{f_{v}^{-1}-f^{-2}} & 1 & 0 \\
\cdots & 0 & 0 & 1 & 0
\end{array}\right) \\
& \widetilde{D}=\left(\begin{array}{ccccc}
\cdots & \cdots & \cdots & \cdots & \cdots \\
\cdots & e^{f_{u}^{-3}-f^{-3}} & -1 & 0 & 0 \\
\cdots & 0 & e^{f_{u}^{-2}-f^{-2}} & -1 & 0 \\
\cdots & 0 & 0 & e^{f_{u}^{-1}-f^{-1}} & 0 \\
\cdots & 0 & 0 & 0 & z_{-v} e^{f_{u,-v}^{-1}-f_{-v}^{-1}}
\end{array}\right) \text {, }
\end{aligned}
$$

а также две матрицы, имеющие только по два нетривиальных элемента,

$$
\tilde{b}=\left(\begin{array}{ccc}
\cdots & \cdots & \cdots \\
\cdots & 0 & 0 \\
\cdots & 0 & -\frac{1}{R} \\
\cdots & 0 & -\frac{z_{-v}}{R_{u}} e^{f_{u,-v}^{-1}-f_{-v}^{-1}}
\end{array}\right), \quad \tilde{d}=\left(\begin{array}{ccc}
\cdots & \cdots & \cdots \\
\cdots & 0 & 0 \\
\cdots & 0 & -\frac{1}{S} \\
\cdots & 0 & -\frac{z_{-v}}{S_{-v}} e^{f_{u,-v}^{-1}-f_{u}^{-1}}
\end{array}\right)
$$

ПРЕДЛОЖЕНИЕ 4. Система уравнений

$$
\begin{aligned}
\widetilde{P}_{-u} & =\tilde{A} \widetilde{P}, & \widetilde{P}_{-v} & =\widetilde{B} \widetilde{P}+\tilde{b} \widetilde{Q}, \\
\widetilde{Q}_{v} & =\widetilde{C} \widetilde{Q}, & \widetilde{Q}_{u} & =\widetilde{D} \widetilde{Q}+\tilde{d} \widetilde{P}
\end{aligned}
$$

совместна тогда и только тогда, когда ее коэфбициенты удовлетворяют полубесконечной цепочке

$$
\begin{aligned}
e^{\Delta f} & =\frac{z_{u,-v}^{-1}}{z}, \\
e^{\Delta f^{j}} & =\frac{z^{j+1}}{z^{j}} \quad \text { npu } \quad-\infty<j \leqslant-1 .
\end{aligned}
$$




\section{4. КОНЕЧНЫЕ ЦЕПОЧКИ}

Замкнем полубесконечную цепочку (33), (34) путем наложения дополнительного (вырожденного) краевого условия $e^{f^{N+1}}=0$. Полученная цепочка совпадает с цепочкой (4), приведенной в разделе 1. При этом можно замкнуть также и пару Лакса, полагая $y^{N+1}=0, \psi^{N+1}=0$. Найденная пара запишется, очевидно, в виде матричной системы

$$
\begin{aligned}
P_{-u} & =A P+a Q, & P_{-v} & =B P, \\
Q_{v} & =C Q+c P, & Q_{u} & =D Q .
\end{aligned}
$$

с матричными коэффициентами размера $(N+2) \times(N+2)$ следующего вида:

$$
\begin{aligned}
& A=\left(\begin{array}{ccccccc}
z_{u} & 0 & 0 & \cdots & 0 & 0 & 0 \\
0 & 1 & 0 & \cdots & 0 & 0 & 0 \\
0 & e^{f_{-u}^{1}-f} & 1 & \cdots & 0 & 0 & 0 \\
\cdots & \cdots & \cdots & \cdots & \cdots & \cdots & \cdots \\
0 & 0 & 0 & \cdots & e^{f_{-u}^{N-1}-f^{N-2}} & 1 & 0 \\
0 & 0 & 0 & \cdots & 0 & e^{f_{-u}^{N}-f^{N-1}} & 1
\end{array}\right) \\
& B=\left(\begin{array}{cccccc}
0 & 1 & 0 & \cdots & 0 & 0 \\
0 & e^{f_{-v}-f} & -1 & \cdots & 0 & 0 \\
0 & 0 & e^{f_{-v}^{1}-f^{1}} & \cdots & 0 & 0 \\
\cdots & \cdots & \cdots & \cdots & \cdots & \cdots \\
0 & 0 & 0 & \cdots & e^{f_{-v}^{N-1}-f^{N-1}} & -1 \\
0 & 0 & 0 & \cdots & 0 & e^{f_{-v}^{N}-f^{N}}
\end{array}\right), \\
& C=\left(\begin{array}{ccccccc}
z_{v} & 0 & 0 & \cdots & 0 & 0 & 0 \\
0 & 1 & 0 & \cdots & 0 & 0 & 0 \\
0 & e^{f_{v}^{1}-f} & 1 & \cdots & 0 & 0 & 0 \\
\cdots & \cdots & \cdots & \cdots & \cdots & \cdots & \cdots \\
0 & 0 & 0 & \cdots & e^{f_{v}^{N-1}-f^{N-2}} & 1 & 0 \\
0 & 0 & 0 & \cdots & 0 & e^{f_{v}^{N}-f^{N-1}} & 1
\end{array}\right) \text {, } \\
& D=\left(\begin{array}{cccccc}
0 & 1 & 0 & \cdots & 0 & 0 \\
0 & e^{f_{u}-f} & -1 & \cdots & 0 & 0 \\
0 & 0 & e^{f_{u}^{1}-f^{1}} & \cdots & 0 & 0 \\
\cdots & \cdots & \cdots & \cdots & \cdots & \cdots \\
0 & 0 & 0 & \cdots & e^{f_{u}^{N-1}-f^{N-1}} & -1 \\
0 & 0 & 0 & \cdots & 0 & e^{f_{u}^{N}-f^{N}}
\end{array}\right) .
\end{aligned}
$$

Матрицы $a$ и $b$ также имеют размер $(N+2) \times(N+2)$. Все элементы этих матриц равны нулю, кроме двух первых элементов первого столбца: $a_{00}=X_{-u, v} z_{-u}, a_{10}=$ $X_{-u}, \quad c_{00}=H_{-u, v} z_{v}, \quad c_{10}=H_{v}$.

Если наложить условие обрыва (15) на оба конца цепочки, то получится система 
уравнений

$$
\begin{aligned}
e^{\Delta f^{-1}} & =\frac{z}{z_{-u, v}^{1}}, \\
e^{\Delta f^{j}} & =\frac{z^{j+1}}{z^{j}} \quad \text { при } \quad 0 \leqslant j \leqslant N-1, \\
e^{\Delta f^{N}} & =\frac{z_{u,-v}^{N-1}}{z^{N}}
\end{aligned}
$$

(см. систему (6) при $z=1+h e^{f_{v}-f_{u}^{-1}}$ и $h=1$ ). Пользуясь правилом обрыва линейных цепочек пары Лакса, рассмотренным выше, легко можно выписать пару Лакса для системы (60). Нетрудно проверить, что она будет иметь вид

$$
\begin{aligned}
\widehat{P}_{-u} & =\hat{A} \widehat{P}+\lambda \hat{a} \widehat{Q}, & \widehat{P}_{-v} & =\widehat{B} \widehat{P}+\hat{b} \widehat{Q}, \\
\widehat{Q}_{v} & =\widehat{C} \widehat{Q}+\lambda^{-1} \hat{c} \widehat{P}, & \widehat{Q}_{u} & =\widehat{D} \widehat{Q}+\hat{d} \widehat{P},
\end{aligned}
$$

где $\lambda$ - спектральный параметр, а матрицы коэффициентов заданы как

$$
\begin{aligned}
& \hat{A}=\left(\begin{array}{cccccccc}
z_{u} & 0 & 0 & \cdots & 0 & 0 & 0 & 0 \\
0 & 1 & 0 & \cdots & 0 & 0 & 0 & 0 \\
0 & e^{f_{-u}^{1}-f} & 1 & \cdots & 0 & 0 & 0 & 0 \\
\cdots & \cdots & \cdots & \cdots & \cdots & \cdots & \cdots & \cdots \\
0 & 0 & 0 & \cdots & 1 & 0 & 0 & 0 \\
0 & 0 & 0 & \cdots & e^{f_{-u}^{N-2}-f^{N-3}} & 1 & 0 & 0 \\
0 & 0 & 0 & \cdots & 0 & e^{f_{-u}^{N-1}-f^{N-2}} & 1 & 0 \\
0 & 0 & 0 & \cdots & 0 & 0 & 1 & 0
\end{array}\right) \\
& \widehat{B}=\left(\begin{array}{ccccccc}
0 & 1 & 0 & \cdots & 0 & 0 & 0 \\
0 & e^{f_{-v}-f} & -1 & \cdots & 0 & 0 & 0 \\
0 & 0 & e^{f_{-v}^{1}-f^{1}} & \cdots & 0 & 0 & 0 \\
\cdots & \cdots & \cdots & \cdots & \cdots & \cdots & \cdots \\
0 & 0 & 0 & \cdots & -1 & 0 & 0 \\
0 & 0 & 0 & \cdots & e^{f_{-v}^{N-2}-f^{N-2}} & -1 & 0 \\
0 & 0 & 0 & \cdots & 0 & e^{f_{-v}^{N-1}-f^{N-1}} & 0 \\
0 & 0 & 0 & \cdots & 0 & 0 & z_{-v}^{N} e^{f_{u, v}^{N-1}-f_{u}^{N-1}}
\end{array}\right), \\
& \widehat{C}=\left(\begin{array}{cccccccc}
z_{v} & 0 & 0 & \cdots & 0 & 0 & 0 & 0 \\
0 & 1 & 0 & \cdots & 0 & 0 & 0 & 0 \\
0 & e^{f_{v}^{1}-f} & 1 & \cdots & 0 & 0 & 0 & 0 \\
\cdots & \cdots & \cdots & \cdots & \cdots & \cdots & \cdots & \cdots \\
0 & 0 & 0 & \cdots & 1 & 0 & 0 & 0 \\
0 & 0 & 0 & \cdots & e^{f_{v}^{N-2}-f^{N-3}} & 1 & 0 & 0 \\
0 & 0 & 0 & \cdots & 0 & e^{f_{v}^{N-1}-f^{N-2}} & 1 & 0 \\
0 & 0 & 0 & \cdots & 0 & 0 & 1 & 0
\end{array}\right)
\end{aligned}
$$




$$
\begin{aligned}
& \widehat{D}=\left(\begin{array}{ccccccc}
0 & 1 & 0 & \cdots & 0 & 0 & 0 \\
0 & e^{f_{u}-f} & -1 & \cdots & 0 & 0 & 0 \\
0 & 0 & e^{f_{u}^{1}-f^{1}} & \cdots & 0 & 0 & 0 \\
\cdots & \cdots & \cdots & \cdots & \cdots & \cdots & \cdots \\
0 & 0 & 0 & \cdots & -1 & 0 & 0 \\
0 & 0 & 0 & \cdots & e^{f_{u}^{N-2}-f^{N-2}} & -1 & 0 \\
0 & 0 & 0 & \cdots & 0 & e^{f_{u}^{N-1}-f^{N-1}} & 0 \\
0 & 0 & 0 & \cdots & 0 & 0 & z_{-v}^{N} e^{f_{u,-v}^{N-1}-f_{-v}^{N-1}}
\end{array}\right), \\
& \hat{a}=\left(\begin{array}{cccc}
X_{-u, v} z_{-u} & 0 & \ldots & 0 \\
X_{-u} & 0 & \ldots & 0 \\
0 & 0 & \ldots & 0 \\
\ldots & \ldots & \ldots & \ldots \\
0 & 0 & \ldots & 0
\end{array}\right), \quad \hat{c}=\left(\begin{array}{cccc}
H_{-u, v} z_{v} & 0 & \ldots & 0 \\
H_{v} & 0 & \ldots & 0 \\
0 & 0 & \ldots & 0 \\
\ldots & \ldots & \ldots & \ldots \\
0 & 0 & \ldots & 0
\end{array}\right) \\
& \hat{b}=\left(\begin{array}{cccc}
0 & \cdots & 0 & 0 \\
\cdots & \cdots & \cdots & 0 \\
0 & \cdots & 0 & -\frac{1}{R^{N}} \\
0 & \cdots & 0 & -\frac{z_{-v}^{N}}{R_{u}^{N}} e^{f_{u-v}^{N-1}-f_{-v}^{N-1}}
\end{array}\right) \\
& \hat{d}=\left(\begin{array}{cccc}
0 & \cdots & 0 & 0 \\
\cdots & \cdots & \cdots & \cdots \\
0 & \cdots & 0 & -\frac{1}{S^{N}} \\
0 & \cdots & 0 & -\frac{z_{-v}^{N}}{S_{-v}^{N}} e^{f_{u-v}^{N-1}-f_{u}^{N-1}}
\end{array}\right)
\end{aligned}
$$

где функции $R^{N}$ и $S^{N}$ определяются из уравнений

$$
\begin{gathered}
R_{u}^{N}=R^{N} z_{-v}^{N} e^{f_{u,-v}^{N-1}-f_{-v}^{N-1}}, \quad R_{v}^{N}=R^{N} z_{-u}^{N} e^{f_{-u}^{N}-f_{-u, v}^{N},} \\
S_{u}^{N}=S^{N} \frac{e^{f^{N}-f_{u}^{N}}}{z_{-u,-v}^{N-1}}, \quad S_{v}^{N}=S^{N} \frac{e^{f_{v}^{N-1}-f^{N-1}}}{z^{N-1}}, \\
R_{u v}^{N} S^{N}=\frac{z^{N}}{z^{N}-1} .
\end{gathered}
$$

Поясним, как появляется спектральный параметр в системе (61), (62). Очевидно, что каждая из функций $H$ и $X$ определяется уравнениями $(23),(24)$ лишь с точностью до постоянного множителя. С учетом равенства (22) остается ровно один произвольный множитель, который можно принять за спектральный параметр.

Благодарности. Работа была поддержана РФФИ (гранты № 04-01-00190, 05-0100775). 


\section{Список литературы}

[1] R. Hirota. J. Phys. Soc. Japan. 1981. V. 50. P. 3785.

[2] D. Levi, L. Pilloni, P. M. Santini. J. Phys. A. 1981. V. 14. P. 1567.

[3] G. Darboux. Leçons sur la Théorie Générale des Surfaces et les Applications Géométrique du Calcul Infinitésimal. V. 2. Paris: Goutier-Villars, 1915.

[4] А.Н. Лезнов, М.В. Савельев. Групповые методы интегрирования нелинейных динамических систем. М.: Наука, 1985.

[5] R.S. Ward. Phys. Lett. A. 1995. V. 199. P. 45.

[6] А.В. Забродин. ТМФ. 1997. Т. 113. № 2. С. 179; 1998. Т. 116. № 1. С. 54.

[7] И. Т. Хабибуллин. ТМФ. 2005. Т. 143. № 1. С. 33; I. T. Habibullin. Multidimensional integrable boundary problems. nlin.SI/0401028; Discrete Toda field equations. nlin.SI/0503055.

[8] И. А. Дынников, С.П. Новиков. УМН. 1997. Т. 52. № 5. С. 175.

[9] Ю. Б. Сурис. Алгебра и анализ. 1990. Т. 2. № 2. С. 141.

[10] Т. Г. Казакова. ТМФ. 2004. Т. 138. № 3. С. 422.

Поступила в редакцию 2.IV.2005 г. 